\title{
WEAK CONTINUITY OF INVARIANT MEASURES FOR A CLASS OF PIECEWISE MONOTONIC TRANSFORMATIONS
}

\author{
ABRAHAM BOYARSKY ${ }^{1}$ AND SARAH COOPER
}

\begin{abstract}
Let $\tau_{a}:[0,1] \rightarrow[0,1], 0<a<\frac{1}{2}$, denote the class of transformations defined by

$$
\tau_{a}(x)= \begin{cases}2 x, & 0<x<\frac{1}{2}, \\ (2-a)-2(1-a) x, & \frac{1}{2}<x<1 .\end{cases}
$$

For $a^{*}<a<\frac{1}{2}$, the transformation $\tau_{a}$ admits unique absolutely continuous invariant measures $\mu_{a}$ whose density function $f_{a}(x)$ assumes the value 0 on an interval which expands as $a \uparrow \frac{1}{2}$. Furthermore, the measures $\mu_{a}$ converge weakly to the measure $\mu_{1 / 2}=\frac{1}{2} \delta_{1 / 2}+\frac{1}{2} \delta_{1}$ as $a \uparrow \frac{1}{2}$, where $\mu_{1 / 2}$ is an invariant measure under $\tau_{a}$, and $\delta_{y}$ denotes the Dirac measure at the point $y$.
\end{abstract}

1. Introduction. Let $\tau:[0,1] \rightarrow[0,1]$ be a piecewise $C^{2}$ transformation satisfying $\inf _{x}|d \tau / d x|>1$, where the slope exists. Lasota and Yorke [2] established the existence of absolutely continuous invariant measures for this class of transformations.

The question of continuity of the invariant measures with respect to the transformation itself is considered in [1]. Under certain restrictions, one of which is that the slopes of all segments of $\tau$ be bounded away from 1, Kowalski [1, Theorem 3] gives necessary and sufficient conditions that guarantee: $\tau_{n} \rightarrow \tau$ implies $f_{n} \rightarrow f$, where $f_{n}, f$ are the densities of the measures $\mu_{n}, \mu$ invariant under $\tau_{n}, \tau$, respectively. The convergence of $\tau_{n}$ and $f_{n}$ are both in the $L_{1}$-norm on $[0,1]$.

The object of this note is to give an example of $\tau_{n} \rightarrow \tau$ such that $\mu_{n} \Rightarrow \mu$, where $\tau$ has slope 1 on an interval, $\mu$ is a point measure invariant under $\tau$, and $\Rightarrow$ means weak convergence.

2. Properties of $\left\{\tau_{a}\right\}$. Let $\tau_{a}:[0,1] \rightarrow[0,1]$ denote a class of transformations labelled by $a, 0<a<\frac{1}{2}$, and defined by

$$
\tau_{a}(x)= \begin{cases}2 x, & 0<x<\frac{1}{2}, \\ (2-a)-2(1-a) x, & \frac{1}{2}<x<1 .\end{cases}
$$

For $0<a<\frac{1}{2}$, it follows from [3] that $\tau_{a}$ admits a unique absolutely continuous invariant measure $\mu_{a}$, whose density with respect to the Lebesgue measure is denoted by $f_{a}$.

Received by the editors May 21, 1979.

1980 Mathematics Subject Classification. Primary 28D10; Secondary 39C05.

${ }^{1}$ The research of the first author was supported by a grant from NSERC, Grant No. A-9072. 
Lemma 1. Let $a^{*}=(7-\sqrt{17}) / 8$. For $a^{*}<a<\frac{1}{2}, f_{a}(x)=0$ when $4 a^{2}-5 a+2$ $<x<2 a$.

Proof. Let $0<a<\frac{1}{2}$. Since no orbit $\left\{\tau_{a}^{i}(x)\right\}, x \in(0,1]$ can return to $(0, a)$ after it has left it, the support of $f_{a} \subset[a, 1]$. Let $\varepsilon>0, \delta>0$ and define $I_{a}=[a, a+\varepsilon]$ and $J_{a}=[1-\delta, 1]$. Assume further that $\frac{1}{2}<a+\varepsilon<1-\delta$ and $\tau_{a}(a)<\tau_{a}(a+\varepsilon)$. Then $\tau_{a}\left(I_{a}\right)=[2 a, 1]$ and $\tau_{a}\left(J_{a}\right)=[a,(2-a)-2(1-a)(1-\delta)]$. We now show that there exist $\varepsilon$ and $\delta$ such that $I_{a} \cup J_{a}$ is $\tau_{a}$-invariant. (A set $X$ is $\tau_{a}$-invariant if $\tau_{a}(X)=X$ a.e.)

We impose two conditions: $\tau_{a}\left(I_{a}\right)=J_{a}$ and $\tau_{a}\left(J_{a}\right)=I_{a}$. The first requires that $[2 a, 1]=[1-\delta, 1]$. Thus $\delta=1-2 a$. The second condition requires that $[a$, $(2-a)-2(1-a)(1-\delta)]=[a, a+\varepsilon]$. Thus $\varepsilon=2(1-a)(1-2 a)$. For these values of $\varepsilon$ and $\delta, I_{a} \cup J_{a}$ is $\tau_{a}$-invariant. Since the support of the unique invariant measure $\mu_{a}$ is $\tau_{a}$-invariant, the support of $f_{a}$ is contained in $I_{a} \cup J_{a}$.

The set $I_{a}$ will meet the set $J_{a}$ when $a+\varepsilon=1-\delta$. This occurs when $a+$ $2(1-a)(1-2 a)=2 a$. The roots of this quadratic are $(7 \pm \sqrt{17}) / 8$. Only $a^{*}=$ $(7-\sqrt{17}) / 8$ falls into the interval $[0,1]$. Thus $I_{a^{*}} \cap J_{a^{*}}=\left\{2 a^{*}\right\}$.

For $a^{*}<a<\frac{1}{2}, \varepsilon_{a} \equiv 2(1-a)(1-2 a)<\varepsilon_{a^{*}}$ and $1-\delta_{a} \equiv 2 a>1-\delta_{a^{*}}$. Clearly, $I_{a} \subset I_{a^{*}}$ and $J_{a} \subset J_{a^{*}}$, where the containment is strict. Since the support of $f_{a}$ is contained in $I_{a} \cup J_{a}=\left[a, 4 a^{2}-5 a+2\right] \cup[2 a, 1]$, the conclusion follows. Q.E.D.

From the definition of the Frobenius-Perron Operator [2], it can be easily shown that $f_{a}$ is the solution of the functional equation

$$
f_{a}(x)= \begin{cases}0, & 0<x<a, \\ \frac{1}{2(1-a)} f_{a}\left(\frac{1}{2}+\frac{1-x}{2(1-a)}\right), & a<x<2 a, \\ \frac{1}{2} f_{a}\left(\frac{x}{2}\right)+\frac{1}{2(1-a)} f_{a}\left(\frac{1}{2}+\frac{1-x}{2(1-a)}\right), & 2 a<x<1,\end{cases}
$$

such that

$$
\int_{0}^{1} f_{a}(x) d x=1
$$

Equation (1) provides an alternative proof of Lemma 1, which goes as follows. It can be shown that if $4 a^{2}-5 a+2<x<2 a$, then

$$
4 a^{2}-5 a+2<\frac{1}{2}+(1-x) / 2(1-a)<2 a
$$

where $a^{*}<a<\frac{1}{2}$. For $0<a<\frac{1}{2}, 0<1 / 2(1-a)<1$, and from [2] it follows that $f_{a}$ is bounded. Starting with any point $x$ in the interval $\left(4 a^{2}-5 a+2,2 a\right)$, repeated iterations by $f_{a}$ show that for any $\varepsilon>0, f_{a}(x)<\varepsilon$. Hence, $f_{a}(x)=0$. When $a=a^{*}, f_{a^{*}}\left(2 a^{*}\right)=0$.

Returning to equation (1), let us define $F_{a}(x)=\int_{0}^{x} f_{a}(t) d t$. From (1) and (2), it follows that 


$$
F_{a}(x)= \begin{cases}0, & 0<x<a, \\ 1-F_{a}\left(\frac{1}{2}+\frac{1-x}{2(1-a)}\right), & a<x<2 a, \\ 1+F_{a}\left(\frac{x}{2}\right)-F_{a}\left(\frac{1}{2}+\frac{1-x}{2(1-a)}\right), & 2 a<x<1 .\end{cases}
$$

Define $x_{a}$ to be the number satisfying $x_{a}=\frac{1}{2}+\left(1-x_{a}\right) / 2(1-a)$. Thus $x_{a}=$ $(2-a) /(3-2 a)$. Clearly $x_{a}<2 a$ for these values of $a$. Since $a+\varepsilon_{a}=4 a^{2}-5 a$ $+2<(2-a) /(3-2 a)$ for $(7-\sqrt{17}) / 8<a<\frac{1}{2}$, we get $x_{a} \notin I_{a} \cup J_{a}$. Hence $f_{a}\left(x_{a}\right)=0$. Now, from (3), we have $F_{a}\left(x_{a}\right)=1-F_{a}\left(x_{a}\right)$. Thus, $F_{a}\left(x_{a}\right)=\frac{1}{2}$. Q.E.D.

For the transformation

$$
\tau_{1 / 2}(x)= \begin{cases}2 x, & 0<x<\frac{1}{2}, \\ 3 / 2-x, & \frac{1}{2}<x<1,\end{cases}
$$

there are an uncountable number of point measures invariant under $\tau_{1 / 2}$. Indeed any measure supported on the two points $\{x, 1.5-x\}$, where $\frac{1}{2}<x<1$, is an invariant measure. In particular $\left\{\frac{1}{2}, 1\right\}$ is the support of an invariant measure. Since the orbit going through these two points is a 2-cycle, they must have equal weight. Hence, let $\mu_{1 / 2}=\frac{1}{2} \delta_{1 / 2}+\frac{1}{2} \delta_{1}$. For this invariant measure, we prove

THEOREM 1. $\mu_{a} \Rightarrow \mu_{1 / 2}$ as $a \uparrow \frac{1}{2}$.

Proof. From Lemma 1 , it follows that $I_{a} \downarrow\left\{\frac{1}{2}\right\}$ and $J_{a} \uparrow\{1\}$ as $a \uparrow \frac{1}{2}$. Thus, the supports of $\mu_{a}$ approach the set $\left\{\frac{1}{2}, 1\right\}$ as $a \uparrow \frac{1}{2}$. Let $F_{1 / 2}(x)$ be the distribution function induced by $\mu_{1 / 2}$, i.e.,

$$
F_{1 / 2}(x)= \begin{cases}0, & 0<x<\frac{1}{2}, \\ \frac{1}{2}, & \frac{1}{2}<x<1, \\ 1, & x=1 .\end{cases}
$$

Using $F_{a}\left(x_{a}\right)=\frac{1}{2}$, it follows that $F_{a}(x) \rightarrow F_{1 / 2}(x)$ pointwise for all $x$ except $x=\frac{1}{2}$ and 1 as $a \uparrow \frac{1}{2}$. This is equivalent to the assertion of the theorem. Q.E.D.

Final note. It would be of interest if one could find a class of transformations admitting slope 0 (for example, $\tau_{a}(x)=a x(1-x), 0<a<1$ ), whose invariant measures are weakly continuous with respect to $a$, at least at some $a$. This would be an important step in justifying the use of such discrete models in practice. Numerical results indicate that there is weak continuity of invariant measures from the left for $\tau_{4}(x)=4 x(1-x)$.

\section{REFERENCES}

1. Z. S. Kowalski, Ergodic properties of piecewise monotonic transformations, Soc. Math. de France Astérisque 49 (1977), 145-149.

2. A. Lasota and J. A. Yorke, On the existence of invariant measures for piecewise monotonic transformations, Trans. Amer. Math. Soc. 186 (1973), $481-488$.

3. T.-Y. Li and J. A. Yorke, Engodic transformations from an interval into itself, Trans. Amer. Math. Soc. 235 (1978), 183-192.

Department of Mathematics, Sir George Wiluiams Campus, Concordia University, Montreal, Canada 